\title{
Geometrical and Numerical Approach to Solve Transonic Gas Equation
}

\author{
Sami H. Altoum ${ }^{1,2}$ \\ ${ }^{1}$ AL-Qunfudhah University College, Umm Al-Qura University, Mecca, KSA \\ ${ }^{2}$ Academy of Engineering Sciences, Khartoum, Sudan \\ Email: shtoum@uqu.edu.sa
}

How to cite this paper: Altoum, S.H (2018) Geometrical and Numerical Approach to Solve Transonic Gas Equation. Journal of Applied Mathematics and Physics, 6, 1659-1674.

https://doi.org/10.4236/jamp.2018.68142

Received: July 9, 2018

Accepted: August 12, 2018

Published: August 15, 2018

Copyright $\odot 2018$ by author and Scientific Research Publishing Inc.

This work is licensed under the Creative Commons Attribution International License (CC BY 4.0).

http://creativecommons.org/licenses/by/4.0/

\begin{abstract}
In this paper, the transonic gas equation will be considered. Different methods will be presented and discussed. We will show that there is no unique solution in the computational domain $[-1,1]^{2}$ with Dirichlet boundary conditions.
\end{abstract}

\section{Keywords}

Symmetry Group, Infinitesimal Generator, Prolongation, Transonic Gas, Numerical Solution

\section{Introduction}

Consider the transonic gas equation

$$
u_{x} u_{x x}+u_{y y}=0
$$

The study of gas dynamics is often associated with the flight of modern high speed aircraft and atmospheric reentry of space exploration vehicles; however, its origins lie with simpler machines. At the beginning of the 19th century, investigation into the behavior of fired bullets led to improvement in the accuracy and capabilities of guns and artillery. As the century progressed, inventors such as Gustaf de Laval advanced the field, while researchers such as Ernst Mach sought to understand the physical phenomenon involved through experimentation the model equation gas equation. Lie groups lie at the intersection of two fundamental fields of mathematics: algebra and geometry. A Lie group is first of all a group. Secondly it is a smooth manifold which is a specific kind of geometric object. The circle and the sphere are examples of smooth manifolds. Finally the algebraic structure and the geometric structure must be compatible in a precise way. Informally, a Lie group is a group of symmetries where the symmetries 
are continuous. A circle has a continuous group of symmetries, we can rotate the circle an arbitrarily small amount and it looks the same. This is in contrast to the hexagon, for example. If you rotate the hexagon by a small amount then it will look different. Only rotations that are multiples of one-sixth of a full turn are symmetries of a hexagon. Lie groups were studied by the Norwegian mathematician Sophus Lie at the end of the 19th century. Lie was interested in solving equations. At that time techniques for solving equations were basically a bag of tricks. A typical tool was to make a clever change of variables which would make one of the variables drop out of the equations. Lie's basic insight was that when this happened it was due to an underlying symmetric of the equations, and that underlying this symmetry was what is now called a Lie group. Lie groups are ubiquitous in mathematics and all areas of science. Associated to any system which has a continuous group of symmetries is a Lie group. The symmetry Lie groups and invariant solutions for the wave diffusion in gas and the transonic equation were obtained by Ames, R. J. Lohner [1]. After their work, Torissi and Valenti [2] applied the infinitesimal group analysis to a second-order nonlinear wave equation telling a non-homogeneous manner. Bluman and Kumei [3] deliberate the wave equations with two and four-parameter symmetry groups and the corresponding invariant (similarity) solutions. Peters and Ames [4] applied Lie group method to the nonlinear dynamic equations of elastic string. Group invariant solutions for a second-order hyperbolic wave equation including an axially transported speed and nonlinear terms were constructed by Fung et al. [5]. Ozkaya and Pakdemirli [6] accurate exact solutions of accidental vibrations of a fiber moving with time-dependent velocity. Simple examples of Lie groups contain translation, rotation and scaling. Lie groups and infinitesimal generators can be logically prolonged to act on the space of independent variables [7] [8] [9]. Sami H. Altoum, studied Lie group and RK4 for solving nonlinear first order ODEs [10]. This article is organized as follows: In Section 2 we state the main problem. In Section 3 introduced some preliminary definitions related to Lie group. In Section $4 \mathrm{~A}$ Lie group method approach is purposed to obtain exact solution. In Section 5 we recall a solution obtained see [10]. In Section 6 a pdsolve maple method is proposed as technique to get an analytical solution. In Section 7 a central finite difference method proposed to solve transonic gas equation. In Section 8 a high order finite difference method is proposed as technique to show that there is no unique solution of transonic gas equation. Finally we conclude.

\section{Problem}

Solve

$$
u_{x} u_{x x}+u_{y y}=0 \text { in } \Omega=[-1,1]^{2}
$$

we focus in our study to the homogeneous Dirichlet boundary condition on $\partial \Omega$, when $\partial \Omega$ denotes the boundary of the domain $\Omega$. For simplicity we will use homogeneous Dirichlet boundary condition. Up to now there is now way to 
solve equation manually. So that Lie group technique will be introduced and used as tools to compute a nonzero solution of (2). Furthermore, numerical approach based on central finite difference method will be introduced and a high order accurate finite difference method will be used to show that no unique solution can be found.

\section{Preliminary}

\subsection{Definition of a Group}

Consider invertible transformations in an $n$-dimensional Euclidean space $\mathbb{R}^{n}$ defined by equations of the form

$$
\bar{x}^{i}=f^{i}(x), \quad i=1, \cdots, n,
$$

where $x=\left(x^{1}, \cdots, x^{n}\right) \in \mathbb{R}^{n}$ and $\bar{x}=\left(\bar{x}^{1}, \cdots, \bar{x}^{n}\right) \in \mathbb{R}^{n}$. It is assumed that the vector function $f=\left(f^{1}, \cdots, f^{n}\right)$ is continuous, together with its derivatives. It is also assumed that the coordinates $x^{i}$ and $\bar{x}^{i}$ of points $x$ and $\bar{x}$ are referred to the same coordinate system. Since the above transformation is invertible, there exists the inverse transformation:

$$
x^{i}=\left(f^{-1}\right)^{i}(\bar{x}), i=1, \cdots, n .
$$

We denote $T$ to above transformation and $T^{-1}$ the inverse transformation. Accordingly, $T$ carries any point $x \in \mathbb{R}^{n}$ into a new position $\bar{x} \in \mathbb{R}^{n}$, and $T^{-1}$ returns $\bar{x}$ into the original position $x$. The identical transformation, $\bar{x}^{i}=x^{i}, i=1, \cdots, n$ denoted by $I$. A function $F(x)$ is called an invariant of group $G$ of transformations $\bar{x}^{i}=f^{i}(x, a), i=1, \cdots, n$ if $F$ remains unaltered when one moves along any path curve of the group $G$. In other words, $F$ is an invariant if $F(f(x, a))=F(x)$ identically in $x$ and a in a neighborhood of $a=0$.

\subsection{Infinitesimal Transformation}

Given 1) parameter group $G$ of transformation

$$
\bar{x}^{i}=f^{i}(x, a), i=1, \cdots, n
$$

Let us expand $f^{i}(x, a)$ into Taylor series in the parameter $(a)$ at $a=0$, then invoking the initial condition $\left(f^{i}(x, 0)=x^{i}, i=1, \cdots, n\right)$, we arrive to infinitesimal transformation of $G[10]$.

$$
\bar{x}^{i}=x^{i}+a \xi^{i}(x)=f^{i}(x, 0)+(a-0) \frac{\partial f(x, 0)}{\partial a}+\mathcal{O}\left(a^{2}\right), i=1, \cdots, n
$$

where

$$
\xi^{i}(x)=\frac{\partial}{\partial a}\left[f^{i}(x, a)\right]_{a=0}
$$

Geometrically, the infinitesimal transformation (4) and (5) defines the tangent vector $\xi^{i}(x)=\left(\xi^{1}(x), \cdots, \xi^{n}(x)\right)$ at the point $(0, f(x, 0))$ therefore the tangent vector field of the group $G$ denoted by $\xi^{i}$ and written as a first order li- 
near differential operator

$$
\begin{gathered}
X=\xi^{1}(x) \frac{\partial}{\partial x^{1}}+\cdots+\xi^{n}(x) \frac{\partial}{\partial x^{n}} \\
X=\xi^{i}(x) \frac{\partial}{\partial x^{i}}, i=1, \cdots, n
\end{gathered}
$$

\subsection{System of Differential Equation}

Consider a system of equations in $\mathbb{R}^{n}$

$$
F_{\sigma}(x)=0, \sigma=1, \cdots, s,
$$

where $x$ in $\mathbb{R}^{n}$ and $s<n$. The system (7) is invariant with respect to a group $G$ of transformations (3) [11].

Theorem 1 (cf [11] [12]) The system (7) invariant under group $G$ with infinitesimal generator $X$ if and only if

$$
\left.X F_{\sigma}(x)\right|_{(M)}=0, \sigma=1, \cdots, s
$$

where the symbol $\left.F_{\sigma}\right|_{M}$ means the value $F_{\sigma}$ of on the manifold $M$. The system of (7) is said to be invariant with respect to group $G$ of transformation $\bar{x}^{i}=f^{i}(x, a)$, or that Equation (6) admits $G$ as a symmetry group if $F_{\sigma}(x)=0$, $\sigma=1, \cdots, s$, whenever $x$ solve Equation (7). Geometrically it means that transformations of the group $G$ carry any point of the variety $M$ along this variety. In other words, $\sigma=1, \cdots, s$, the path curve of the group $G$ passing through any point $x \in M$ lies on $M$. Consequently, $M$ is an invariant manifold for $G$.

\subsection{Determining Equations}

Theorem (1) provides the infinitesimal criterion of symmetry group of differential equations. The system of $\mathrm{DE}$ is,

$$
F_{\sigma}\left(x, u, u_{1}, \cdots, u_{k}\right)=0, \sigma=1, \cdots, s
$$

is invariant under the group with an infinitesimal generator $X$ if an only if

$$
\left.X F_{(\sigma)}\right|_{M}=0, \sigma=1, \cdots, s
$$

where $X$ is extended to all derivatives involved in (9). We give three methods to solve transonic gas equation to get exact solutions, the first method using Lie groups, second method using classical solution of PDEs and third method using finite difference.

\subsection{Prolongation}

We need to supplant the slightly hazy notion of a system of D.E. by stiff geometric target specified by evanescence of particular functions. To do this we need to prolong the basic space, representing the independent and dependent variables under consideration, to a space which also represents the various partial derivatives occurring in the system [13]. $f(x)=f\left(x^{1}, \cdots, x^{p}\right)$ of $p$ independent va- 
riables, there are given by:

$$
p_{k}=\left[\begin{array}{c}
p+k+1 \\
k
\end{array}\right],
$$

order partial derivatives of $f$. We derive $k^{\text {th }}$ employ the multi-index notation

$$
\frac{\partial^{k} f}{\partial x^{k}}=\frac{\partial^{k} f}{\partial_{x_{1}}^{j_{1}} \partial_{x_{2}}^{j_{2}} \cdots \partial_{x_{n}}^{j_{n}}} ; j_{1}+j_{2}+\cdots+j_{n}=k
$$

Consider the case $P=2, q=1$ then $X \approx \mathbb{R}^{2}$ has coordinates $\left(x^{1}, x^{2}\right)=(x, y)$ and $U \approx \mathbb{R}$ has the single coordinate $u$. The space $U_{1}$ is isomorphic to $\mathbb{R}^{2}$ with coordinates $\left(u_{x}, u_{y}\right)$, since these represent all the first order partial derivatives of $u$ with respect to $x$ and $y$. Similarly $U_{2} \approx \mathbb{R}^{3}$ has the coordinates $\left(u_{x x}, u_{x y}, u_{y y}\right)$, representing the second order partial derivatives of $u$, and in general $U_{k} \approx \mathbb{R}^{k+1}$, since there are $k+1$ distinct $k-1$ order partial derivatives of $u$. We have the heat equation $u_{t}-u_{x x}=0$. Here, note that there are two independent variables $x, t$, one dependent variable, $u$, so we have the underlying space $X \times U=\mathbb{R}^{3}$ with coordinates $x, u, t$. Since the heat equation contains a second order partial derivative, we consider the prolongation of the 2-jet, namely to the set $X \times U$ which has coordinates $\left(x, t, u, u_{x}, u_{t}, u_{x x}, u_{x t}, u_{t t}\right)$.

\subsection{Lie Algebra of Operator}

A Lie algebras of operators, is a vector space $L$ of operator $X=\xi^{i}(x) \frac{\partial}{\partial x^{i}}$ with the following property. If the operators

$$
X_{1}=\xi_{1}^{i}(x) \frac{\partial}{\partial x^{i}}, X_{2}=\xi_{2}^{i}(x) \frac{\partial}{\partial x^{i}}
$$

are elements of $L$ then their commutator is

$$
\left[X_{1}, X_{2}\right] \equiv X_{1} X_{2}-X_{2} X_{1}=\left(X_{1}\left(\xi_{2}^{i}\right)-X_{2}\left(\xi_{1}^{i}\right)\right) \frac{\partial}{\partial x^{i}}
$$

is bilinear and skew-symmetric also an element of $L$. The dimension $(\operatorname{dim} L)$ of the Lie algebra is the dimension of the vector space $L$. We use the symbol $L_{r}$ to denote an $r$-dimensional Lie algebra. $X \in L_{r}$ is their linear combination, $X=c^{\alpha} X_{\alpha}=\sum_{\alpha=1}^{r} c^{\alpha} X_{\alpha}$ with constant coefficients $c^{\alpha}$. In particular, $\left[X_{\alpha}, X_{\beta}\right] \in L_{r}$, hence

$$
\left[X_{\alpha}, X_{\beta}\right]=c_{\alpha \beta}^{\gamma} X_{\gamma}, \alpha, \beta=1, \cdots, r
$$

\subsection{Main Variables and Total Derivatives}

In differential algebra we deal with an infinite derivatives

$$
x=\left\{x^{i}\right\}, u=\left\{u^{\alpha}\right\}, u_{(1)}=\left\{u_{i}^{\alpha}\right\}, u_{(2)}=\left\{u_{i_{1} i_{2}}^{\alpha}\right\}, u_{(3)}=\left\{u_{i_{1} i_{2} i_{3}}^{\alpha}\right\}, \cdots
$$

where $\alpha=1, \cdots, m ; i, i_{1}, \cdots=1, \cdots, n$. The variables $u_{i i_{2} i_{2}}^{\alpha}, u_{i i_{1} i_{3}}^{\alpha}, \cdots$ are systematic in the subscripts $i_{1} i_{2}, i_{1} i_{2} i_{3}, \cdots$. The main operation in the calculus of differential algebra is the total differentiation given by the following

$$
D_{i}=\frac{\partial}{\partial x^{i}}+u_{i}^{\alpha} \frac{\partial}{\partial u^{\alpha}}+u_{i i_{1}}^{\alpha} \frac{\partial}{\partial u_{i_{1}}^{\alpha}}+u_{i i_{1} i_{2}}^{\alpha} \frac{\partial}{\partial u_{i 1_{2}}^{\alpha}}+\cdots, i=1, \cdots, n
$$


The total derivatives $D_{i}$ act on functions involving any finite number of variables.

$$
D_{i}\left(f\left(x, u, u_{(1)}\right)\right)=\frac{\partial f}{\partial x^{i}}+u_{i}^{\alpha} \frac{\partial f}{\partial u^{\alpha}}+u_{i i_{1}}^{\alpha} \frac{\partial f}{\partial u_{i_{1}}^{\alpha}}
$$

In particular, letting $f=u^{\alpha}, f=u_{j}^{\alpha}, \cdots$ one obtain:

$$
u_{i}^{\alpha}=D_{i}\left(u^{\alpha}\right), u_{i j}^{\alpha}=D_{i}\left(u_{j}^{\alpha}\right)=D_{i} D_{j}\left(u^{\alpha}\right)
$$

Consequently, $x^{i}$ are called independent variables and $u^{\alpha}$ differentiable variables with the successive $u_{(1)}, u_{(2)}, \cdots$

Hence the total derivatives written by:

$$
\begin{aligned}
& D_{x}=\frac{\partial}{\partial x}+u_{x} \frac{\partial}{\partial u}+u_{x x} \frac{\partial}{\partial u_{x}}+u_{x y} \frac{\partial}{\partial u_{y}}+\cdots, \\
& D_{y}=\frac{\partial}{\partial y}+u_{y} \frac{\partial}{\partial u}+u_{x y} \frac{\partial}{\partial u_{x}}+u_{y y} \frac{\partial}{\partial u_{y}}+\cdots
\end{aligned}
$$

We will use a generator of a point transformation group

$$
X_{2}=\xi^{1}(x, y, u) \frac{\partial}{\partial x}+\xi^{2}(x, y, u) \frac{\partial}{\partial y}+\eta(x, y, u) \frac{\partial}{\partial u} .
$$

Extended Equation (14) then $X_{2}$ becomes

$$
\begin{gathered}
X_{2}=\xi^{1} \frac{\partial}{\partial x}+\xi^{2} \frac{\partial}{\partial y}+\eta \frac{\partial}{\partial u}+\varsigma_{1} \frac{\partial}{\partial u_{x}}+\varsigma_{2} \frac{\partial}{\partial u_{y}}+\varsigma_{11} \frac{\partial}{\partial u_{x x}}+\varsigma_{12} \frac{\partial}{\partial u_{x y}}+\varsigma_{22} \frac{\partial}{\partial u_{y y}} \\
\varsigma_{i}^{\alpha}=D_{i}\left(\eta^{\alpha}\right)-u_{j}^{\alpha} D_{i}\left(\xi^{j}\right)
\end{gathered}
$$

and

$$
\begin{aligned}
\varsigma_{i_{1} i_{2}} & =D_{i_{2}}\left(\varsigma_{i_{1}}^{\alpha}\right)-u_{j i_{1}}^{\alpha} D_{i_{2}}\left(\xi^{j}\right) \\
& =D_{i_{2}} D_{i_{1}}\left(\eta^{\alpha}\right)-u_{j}^{\alpha} D_{i_{2}} D_{i_{1}}\left(\xi^{j}\right)-u_{j i_{1}} D_{i_{2}}\left(\xi^{j}\right)
\end{aligned}
$$

then

$$
\begin{aligned}
& \varsigma_{1}=D_{x}(\eta)-u_{x} D_{x}\left(\xi^{1}\right)-u_{y} D_{x}\left(\xi^{2}\right) \\
& \varsigma_{2}=D_{y}(\eta)-u_{x} D_{y}\left(\xi^{1}\right)-u_{y} D_{y}\left(\xi^{2}\right)
\end{aligned}
$$

also we can deduce

$$
\begin{gathered}
\varsigma_{11}=D_{x}\left(\varsigma_{1}\right)-u_{x x} D_{x}\left(\xi^{1}\right)-u_{x y} D_{x}\left(\xi^{2}\right) \\
\varsigma_{12}=D_{y}\left(\varsigma_{1}\right)-u_{x x} D_{y}\left(\xi^{1}\right)-u_{x y} D_{y}\left(\xi^{2}\right) \\
\varsigma_{22}=D_{y}\left(\varsigma_{2}\right)-u_{x y} D_{y}\left(\xi^{1}\right)-u_{y y} D_{y}\left(\xi^{2}\right)
\end{gathered}
$$

then

$$
\begin{gathered}
\varsigma_{1}=\eta_{x}+u_{x} \eta_{u}-u_{x} \xi_{x}^{1}-\left(u_{x}\right)^{2} \xi_{u}^{1}-u_{y} \xi_{x}^{2}-u_{x} u_{y} \xi_{u}^{2} \\
\varsigma_{2}=\eta_{y}+u_{y} \eta_{u}-u_{x} \xi_{y}^{1}-u_{x} u_{y} \xi_{u}^{1}-u_{y} \xi^{2}-\left(u_{y}\right)^{2} \xi_{u}^{2}
\end{gathered}
$$




$$
\begin{gathered}
\varsigma_{11}=\eta_{x x}+2 u_{x} \eta_{x u}+u_{x x} \eta_{u}+\left(u_{x}\right)^{2} \eta_{u u}-2 u_{x x} \xi_{x}^{1}-u_{x} \xi_{x x}^{1}-2\left(u_{x}\right)^{2} \xi_{x u}^{1}-3 u_{x} u_{x x} \xi_{u}^{1} \\
-\left(u_{x}\right)^{3} \xi_{u u}^{1}-2 u_{x y} \xi_{x}^{2}-u_{y} \xi_{x x}^{2}-2 u_{x} u_{y} \xi_{x u}^{2}-\left(u_{y} u_{x x}+2 u_{x} u_{x y}\right) \xi_{u}^{2}-\left(u_{x}\right)^{2} u_{y} \xi_{u u}^{2} \\
\varsigma_{12}=\eta_{x y}+u_{y} \eta_{x u}+u_{x} \eta_{y u}+u_{x y} \eta_{u}+u_{x} u_{y} \eta_{u u}-u_{x y}\left(\xi_{x}^{1}+\xi_{y}^{2}\right)-u_{x} \xi_{x y}^{1}-u_{x x} \xi_{y}^{1} \\
-u_{x} u_{y}\left(\xi_{x u}^{1}+\xi_{y u}^{2}\right)-\left(u_{x}\right)^{2} \xi_{y u}^{1}-\left(2 u_{x} u u_{x y}+u_{y} u_{x x}\right) \xi_{u}^{1}-\left(u_{x}\right)^{2} u_{y} \xi_{u u}^{1} \\
\quad-u_{y} \xi_{x y}^{2}-u_{y y} \xi_{x}^{2}-\left(u_{y}\right)^{2} \xi_{x u}^{2}-\left(2 u_{y} u_{x y}+u_{x} u_{y y}\right) \xi_{u}^{2}-u_{x}\left(u_{y}\right)^{2} \xi_{u u}^{2} \\
\varsigma_{22}=\eta_{y y}+2 u_{y} \eta_{y u}+u_{y y} \eta_{u}+\left(u_{y}\right)^{2} \eta_{u u}-2 u_{y y} \xi_{y}^{2}-u_{y} \xi_{y y}^{2}-2\left(u_{y}\right)^{2} \xi_{y u}^{2}-3 u_{y} u_{y y} \xi_{u}^{2} \\
-\left(u_{y}\right)^{3} \xi_{u u}^{2}-2 u_{x y} \xi_{y}^{1}-u_{x} \xi_{y y}^{1}-2 u_{x} u_{y} \xi_{y u}^{1}\left(u_{x} u_{y y}+2 u_{y} u_{x y}\right) \xi_{u}^{1}-u_{x}\left(u_{y}\right)^{2} \xi_{u u}^{1}
\end{gathered}
$$

\section{Lie Group}

We introduce a geometrical method based on Lie group technique to solve transonic gas equation, namely problem (2).

Considering symmetry operator in the form (15) with unknown coefficients $\xi^{i}$ and $\eta$ to be found the determining Equation (10)

$$
\begin{gathered}
X F=0 \\
X\left(u_{x} u_{x x}+u_{y y}\right)=0 \\
u_{x x} \zeta_{1}+u_{x} \zeta_{11}+\zeta_{22}=0,
\end{gathered}
$$

where we substitute $\varsigma_{1}, \varsigma_{11}$ and $\varsigma_{22}$ see (23), (25), (27) from extended generators calculation of symmetries of PDEs, with two independent variables and set $u_{y y}=-u_{x} u_{x x}$. Then Equation (29) contain the variables $x, y, u, u_{x}, u_{y}, u_{x x} u_{y y}$ whereas $\xi^{1}, \xi^{2}$ and $\eta$ depend only upon $x, y$ and $u$. Accordingly we isolate the terms containing $u_{x y}, u_{x x}, u_{x}, u_{y}$ and those free of these variables, and set each term equal to zero. So, the terms containing $u_{x y}$, lead to following equation

$$
\xi_{y}^{1}+u_{x} \xi_{x}^{2}+u_{y} \xi_{u}^{1}\left(u_{x}\right)^{2} \xi_{u}^{2}=0
$$

whence

$$
\xi_{y}^{1}=0, \xi_{u}^{1}=0, \xi_{x}^{2}=0, \xi_{u}^{2}=0 .
$$

The same argument applied to the terms containing $u_{x x}$ yields

$$
\eta_{x}=0, \eta_{u}-3 \xi_{x}^{1}+2 \xi_{y}^{2}=0
$$

then Equation (29) reduces to

$$
\eta_{y y}=0,2 \eta_{y u}-\xi_{y y}^{2}=0 .
$$

Thus Equation (29) is split into the over determined system of linear PDEs (30) $\&$ (32). The computation shows that the general solution of this system is given by:

$$
\begin{aligned}
& \xi^{1}=C_{1} x+C_{2} \\
& \xi^{2}=C_{3} y+C_{4}
\end{aligned}
$$




$$
\eta=\left(3 C_{1}-2 C_{3}\right) u+C_{5} y+C_{6} .
$$

Finally we can deduce the solution $u$ from Equation (31)

$$
\begin{gathered}
\left(3 C_{1}-2 C_{3}\right) u+C_{5} y+C_{6}=3\left(C_{1} x+C_{2}\right)-2\left(C_{3} y+C_{4}\right) \\
u(x, y)=\frac{\left(C_{1} x+C_{2}\right)-2\left(C_{3} y+C_{4}\right)-C_{5} y-C_{6}}{\left(3 C_{1}-2 C_{3}\right)}
\end{gathered}
$$

we can write $u$ after simplification

$$
u(x, y)=\alpha x+\beta y+\gamma
$$

where

$$
\begin{gathered}
\alpha=\frac{C_{1}}{3 C_{1}-2 C_{3}} \\
\beta=\frac{2 C_{3}+C_{5}}{3 C_{1}-2 C_{3}} \\
\gamma=\frac{C_{2}}{3 C_{1}-2 C_{3}}+\frac{C_{4}}{3 C_{1}-2 C_{3}}-\frac{C_{6}}{3 C_{1}-2 C_{3}}
\end{gathered}
$$

So we have six arbitrary constants $C_{i}$. Thus we arrive at the six-dimensional Lie algebra $L_{6}$ spanned by the operators.

$$
\begin{aligned}
& X_{1}=\frac{\partial}{\partial x}, X_{2}=\frac{\partial}{\partial y}, X_{3}=\frac{\partial}{\partial u}, X_{4}=y \frac{\partial}{\partial u}, \\
& X_{5}=x \frac{\partial}{\partial x}+3 u \frac{\partial}{\partial u}, X_{6}=y \frac{\partial}{\partial y}-2 u \frac{\partial}{\partial u} .
\end{aligned}
$$

Thus, $L_{6}$ is the linear span of the operators (33), $L_{6}=\left\langle X_{1}, \cdots, X_{6}\right\rangle$, from (11), the commutators $\left[X_{\alpha}, X_{\beta}\right]$, of the basic operators and satisfy (12) for a Lie algebra. The result becomes directly visual if the commutators are disposed as in the following Table 1.

\section{Analytical Solution}

The exact solution of non-linear PDEs, (2), given by see [10].

$$
u_{x} u_{x x}+u_{y y}=0 .
$$

Table 1. Lie algebra operators.

\begin{tabular}{ccccccc}
\hline & $X_{1}$ & $X_{2}$ & $X_{3}$ & $X_{4}$ & $X_{5}$ & $X_{6}$ \\
\hline$X_{1}$ & 0 & 0 & 0 & 0 & $X_{1}$ & 0 \\
$X_{2}$ & 0 & 0 & 0 & $X_{3}$ & 0 & $X_{2}$ \\
$X_{3}$ & 0 & 0 & 0 & 0 & $3 X_{3}$ & $-2 X_{3}$ \\
$X_{4}$ & 0 & $-X_{3}$ & 0 & 0 & $3 X_{4}$ & $-3 X_{4}$ \\
$X_{5}$ & $-X_{1}$ & 0 & $-3 X_{3}$ & $-3 X_{4}$ & 0 & 0 \\
$X_{6}$ & 0 & $-X_{2}$ & $2 X_{3}$ & $3 X_{4}$ & 0 & 0 \\
\hline
\end{tabular}


The solution will be:

1) Let $u(x, t) \quad u$ is the solution of (2). Then the function.

$$
u_{1}=c_{1}^{-3} c_{2}^{2} u\left(c_{1} x+c_{3}, c_{2} y+c_{4}\right)+c_{5} y+c_{6},
$$

where $c_{1}, \cdots, c_{6}$ are arbitrary constant, is also a solution.

2) Solutions

$$
\begin{gathered}
u(x, y)=c_{1} x y+c_{2} x+c_{3} y+c_{4} \\
u(x, y)=\frac{\left(x+c_{1}\right)^{2}}{3 a\left(x+c_{1}\right)^{3}}+c_{3} y+c_{4} \\
u(x, y)=\frac{a^{2} c_{1}^{3}}{39}(y+A)^{13}+\frac{2}{3} a(y+A)^{8}(x+B) \\
+3 c_{1}(y+A)^{3}(x+B)^{2}-\frac{(x+B)^{3}}{3 a(y+A)^{2}} \\
u(x, t)=\frac{a^{2} c_{1}^{3}}{39}(y+A)^{13}+\frac{2}{3} a(y+A)^{8}(x+B) \\
+3 c_{1}(y+A)^{3}(x+B)^{2}-\frac{(x+B)^{3}}{3 a(y+A)^{2}} \\
u(x, y)=-a A^{3} y^{2}-\frac{B^{2}}{a A^{2}} x+c_{1}+c_{2} \pm \frac{4}{3}\left(A x+B y+c_{3}\right)^{\frac{3}{2}} \\
u(x, y)=\frac{1}{3}(A y+B)\left(2 c_{1} x+c_{2}\right)^{\frac{3}{2}}-\frac{a c_{1}^{3}}{12 A^{2}}(A y+B)^{4}+c_{3} y+c_{4} \\
u(x, y)=-\frac{3}{7} a A^{2}\left(y+c_{1}\right)^{7}+4 A\left(x+c_{2}\right)^{\frac{3}{2}}\left(y+c_{1}\right)^{\frac{5}{2}}-\frac{\left(x+c_{2}\right)^{3}}{3 a\left(y+c_{1}\right)^{2}}+c_{3} y+c_{4} \\
y(x, y)=-\frac{9 a A^{2}}{y+c_{1}}+4 A\left(\frac{x+c_{2}}{y+c_{1}}\right)^{\frac{3}{2}}-\frac{\left(x+c_{2}\right)^{3}}{3 a\left(y+c_{1}\right)^{2}}+c_{3} y+c_{4} \\
u(y)
\end{gathered}
$$

\section{Pdsolve Maple Method}

The first and second method proposed to study more precisely to find the solution of transonic gas equation. The pdsolve routine usually introduces new functions to express the solution for the indeterminate function. The examples below illustrate the use of pdsolve in solving a single PDE. For examples related to solve the transonic gas equation. Any arbitrary constants introduced while separating the variables are represented as $C_{1}, C_{2}$ and are global general solution of the second order PDE (transonic gas equation) is

$$
u(x, y)=\frac{1}{2} c_{2} y^{2}+C_{1} y+C_{2}-\frac{2}{3} \sqrt{2} \sqrt{-C_{3} c_{2}-x c_{2}} C_{3}-\frac{2}{3} \sqrt{2} \sqrt{-C_{3} c_{2}-x c_{2}} x+C_{4} .
$$

The following is the solution computed by pdsolve automatically. The approach used in this example serves also to address nonlinear equations of the form (2) with homogenous boundary conditions. This document describes how 
pdsolve can automatically adjust the arbitrary functions and constants entering the solution. In order, to determine the coefficient involved in (35) we will introduce some boundary conditions on $\partial \Omega$, where $\Omega$ is the domain of computation. At first step: Let us, use Dirichlet homogeneous boundary condition and $\Omega=[0,1]^{2}$. The system of equation obtained after applying the boundary conditions is:

$$
\begin{gathered}
C_{2}-\frac{2}{3} \sqrt{2} \sqrt{-C_{3} c_{2}-x c_{2}} C_{3}-\frac{2}{3} \sqrt{2} \sqrt{-C_{3} c_{2}-x c_{2} x}+C_{4}=0 \\
\frac{1}{2} c_{2}+C_{1}+C_{2}-\frac{2}{3} \sqrt{2} \sqrt{-C_{3} c_{2}-x c_{2}} C_{3}-\frac{2}{3} \sqrt{2} \sqrt{-C_{3} c_{2}-x c_{2}} x+C_{4}=0 \\
\frac{1}{2} c_{2} y^{2}+C_{1} y+C_{2}-\frac{2}{3} \sqrt{2} \sqrt{-C_{3} c_{2}} C_{3}+C_{4}=0 \\
\frac{1}{2} c_{2} y^{2}+C_{1} y+C_{2}-\frac{2}{3} \sqrt{2} \sqrt{-C_{3} c_{2}-c_{2}} C_{3}-\frac{2}{3} \sqrt{2} \sqrt{-C_{3} c_{2}-c_{2}}+C_{4}=0
\end{gathered}
$$

this system is solved using Maple 18, we get the following result:

- Trivial Solution: in case

$$
\left\{\begin{array}{l}
C_{1}=0 \\
C_{2}=-C_{4} \\
C_{3}=C_{3} \in \mathbb{R} \\
C_{4}=0
\end{array}\right.
$$

we remark $\forall C_{3} \in \mathbb{R}$ we have null solution $u=0$.

- Non-Trivial Solution:

$C_{1}=-\frac{4}{9} \frac{\frac{2}{3} i \sqrt{2}\left(\gamma_{1}\right)(-1 / 2+i / 6 \sqrt{3})-12\left(-\frac{1}{2}+i / 6 \sqrt{3}\right) x^{2}-4 x^{3}+12\left(-\frac{1}{2}+i / 6 \sqrt{3}\right) x+4 x}{y^{2}\left(y^{2}-2 y+1\right)}$

where

$$
\begin{aligned}
\gamma_{1}=i \sqrt{2}+\sqrt{2} \sqrt{3}-2 \sqrt{9 x^{3}-3 i x^{3} \sqrt{3}+1+i \sqrt{3}-9 x^{2}+9 i x^{2} \sqrt{3}-6 i x \sqrt{3}} \\
C_{2}=\beta_{1}\left(\left(\beta_{5}-1 / 2+i / 6 \sqrt{3}\right)-48\left(-\frac{1}{2}+i / 6 \sqrt{3}\right) x^{2}+9 C_{4} y^{2}-16 x^{3}\right. \\
\left.+48(-1 / 2+i / 6 \sqrt{3}) x-9 C_{4} y+16 x\right)
\end{aligned}
$$

where

$$
\begin{gathered}
\beta_{1}=-\frac{1}{9} \frac{1}{y(y-1)} \\
\beta_{2}=2 \sqrt{9 x^{3}-3 i x^{3} \sqrt{3}+1+i \sqrt{3}-9 x^{2}+9 i x^{2} \sqrt{3}-6 i x \sqrt{3}}\left(-\frac{1}{2}+i / 6 \sqrt{3}\right) \\
-12(-1 / 2+i / 6 \sqrt{3}) x^{2} \\
\beta_{3}=(-1+i / 3 \sqrt{3})\left(\frac{2}{3} i \sqrt{2}\left(i \sqrt{2}+\sqrt{2} \sqrt{3}-\beta_{2}\right)-4 x^{3}+12\left(-\frac{1}{2}+i / 6 \sqrt{3}\right) x+4 x\right)
\end{gathered}
$$




$$
\begin{gathered}
\beta_{4}=-4 \sqrt{-\frac{\beta_{3}}{y^{2}\left(y^{2}-2 y+1\right)}} \sqrt{2}(-1 / 2+i / 6 \sqrt{3}) y^{2} \\
+4 \sqrt{2} \sqrt{-\frac{\beta_{2}}{y^{2}\left(y^{2}-2 y+1\right)}}\left(-\frac{1}{2}+i / 6 \sqrt{3}\right) y \\
\beta_{5}=\beta_{4}+\frac{8}{3} i \sqrt{2}\left(i \sqrt{2}+\sqrt{2} \sqrt{3}-2 \sqrt{9 x^{3}-3 i x^{3} \sqrt{3}+1+i \sqrt{3}-9 x^{2}+9 i x^{2} \sqrt{3}-6 i x \sqrt{3}}\right) \\
c_{2}=\frac{\frac{16}{3} i \sqrt{2}\left(i \sqrt{2}+\sqrt{2} \sqrt{3}-2 \sqrt{9 x^{3}-3 i x^{3} \sqrt{3}+1+i \sqrt{3}-9 x^{2}+9 i x^{2} \sqrt{3}-6 i x \sqrt{3}}\right) \alpha_{1}}{9 y^{2}\left(y^{2}-2 y+1\right)}
\end{gathered}
$$

where

$$
\begin{gathered}
\alpha_{1}=\left(-\frac{1}{2}+i / 6 \sqrt{3}\right)-96\left(-\frac{1}{2}+i / 6 \sqrt{3}\right) x^{2}-32 x^{3}+96\left(-\frac{1}{2}+i / 6 \sqrt{3}\right) x+32 x \\
C_{3}=-\frac{1}{2}+i \frac{1}{6} \sqrt{3} .
\end{gathered}
$$

Then the nontrivial solution is

$u(x, y)$

$$
=\frac{2 \sqrt{2}}{27}\left(\delta_{1} \sqrt{\frac{\left(-\frac{1}{3} \sqrt{2}\left(i+\frac{1}{3} \sqrt{3}\right) \delta_{2}+\left(\frac{4}{9} i+i x(x-1)\right) \sqrt{3}+2 x^{3}-3 x^{2}+x\right)(i \sqrt{3}+6 x-3)}{y^{2}(y-1)^{2}}}+\delta_{3}\right)
$$

where

$$
\begin{gathered}
\delta_{1}=\frac{2}{9}\left(-3 / 2 i \sqrt{3}-9 x+\frac{9}{2}\right) \sqrt{2} \sqrt{3} \\
\delta_{2}=\sqrt{\left(-3 i x^{3}+i+9 i x^{2}-6 i x\right) \sqrt{3}+9 x^{3}-9 x^{2}+1} \\
\delta_{3}=\frac{1}{3}(i \sqrt{3}-3) \sqrt{2} \sqrt{3} \sqrt{\frac{\left(\delta_{4}+\left(\frac{4}{9} i+i x(x-1)\right) \sqrt{3}+2 x^{3}-3 x^{2}+x\right)(i \sqrt{3}-3)}{y^{2}(y-1)^{2}}} \\
\delta_{4}=-\frac{1}{3} \sqrt{2}\left(i+\frac{1}{3} \sqrt{3}\right) \sqrt{\left(-3 i x^{3}+i+9 i x^{2}-6 i x\right) \sqrt{3}+9 x^{3}-9 x^{2}+1}
\end{gathered}
$$

Figure 1 presents the shape of curve of transonic gas equation.

\section{Central Finite Difference Method}

Let us consider the two-dimensional nonlinear PDE equations:

$$
u_{x} u_{x x}+u_{y y}=0 \text {, }
$$

and the boundary conditions being:

$$
u(x, 0)=0, u(x, 1)=0, u(0, y)=0, u(1, y)=0 .
$$

The finite difference schemes take the form 


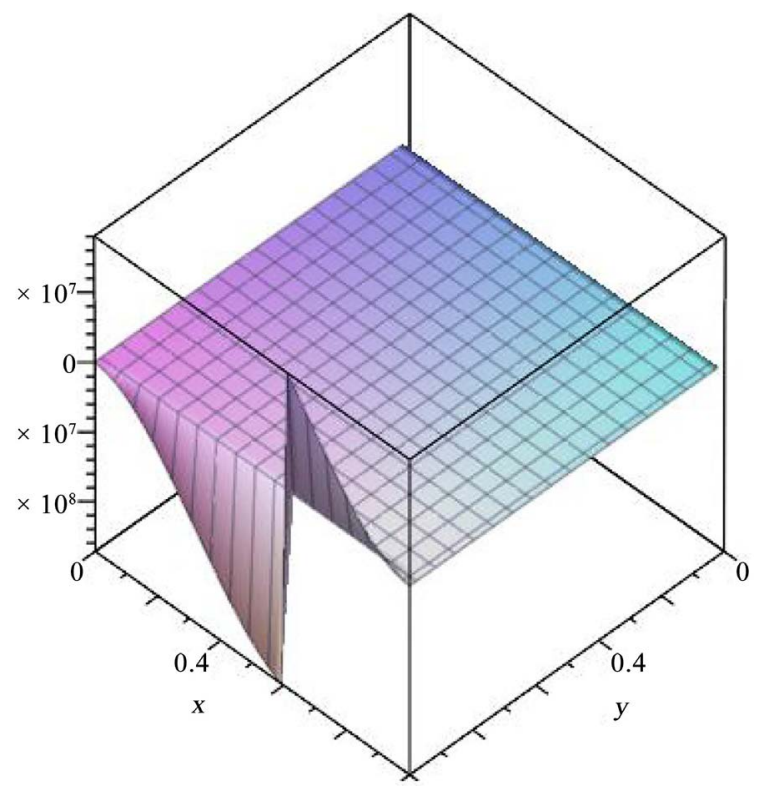

Figure 1. Pdsolve curve of transonic gas equation.

$$
\begin{aligned}
& {\left[\frac{u\left(x_{i+1}, y_{i}\right)-u\left(x_{i}, y_{j}\right)}{\Delta x}\right]\left[\frac{u\left(x_{i+1}, y_{j}\right)-2 u\left(x_{i}, y_{j}\right)+u\left(x_{i}+y_{j}\right)}{(\Delta x)^{2}}\right]} \\
& +\frac{\left[u\left(x_{i}, y_{j+1}\right)-2 u\left(x_{i}, y_{j}\right)+u\left(x_{i}, y_{j-1}\right)\right]}{(\Delta y)^{2}}=0
\end{aligned}
$$

Let $\Delta x=\Delta y=h$

$$
\begin{aligned}
& \left(u\left(x_{i+1}, y_{j}\right)-u\left(x_{i}, y_{j}\right)\right)\left(u\left(x_{i+1}, y_{j}\right)-2 u\left(x_{i}, y_{j}\right)+u\left(x_{i-1}, y_{j-1}\right)\right) \\
& +h\left(u_{i, j+1}-2 u_{i, j}+u_{i, j-1}\right)=0
\end{aligned}
$$

Figure 2 presents central finite difference solution of transonic gas equation.

Figure 3 presents the interior points of solution of transonic gas equation.

\section{High Finite Difference Method}

The key step in solving our PDE numerically using finite difference methods is to replace the derivatives with so-called "finite difference Method". Here is an example of the centered finite difference stencil for the second derivative:

$$
\frac{\partial^{2}}{\partial x^{2}} u(x, y)=\sum_{i=-2}^{2} \beta_{i} u(x+i h, y)+\text { Error }
$$

The stencil is "centered" because $u$ is evaluated at an equal number of points to the right and left of the point where we want to approximate the derivative. The coefficients $\beta_{i}$ are specified such that error is $O\left(h^{6}\right)$. That is, we solve for error, expand in a Taylor series in $h$ and then we choose the coefficients such that the first 5 terms in the Taylor series vanish, i.e. Error $=O\left(h^{5}\right)$ if and only if: 


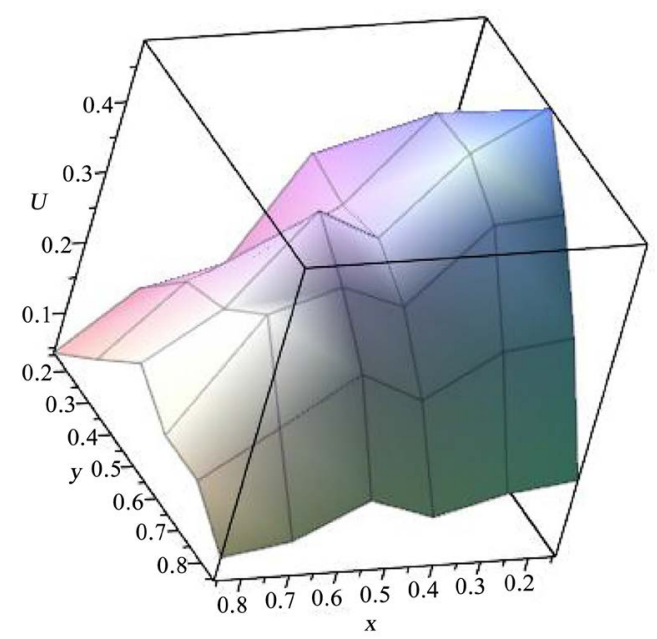

Figure 2. Central finite difference solution.

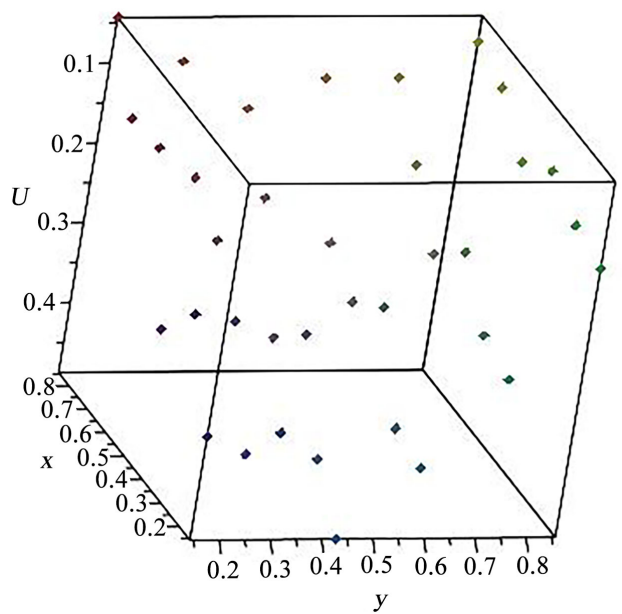

Figure 3. Interior points solution.

$$
\left\{\begin{array}{l}
2 \beta_{-2}-\beta_{1}+\beta_{-1}-2 \beta_{2}=0 \\
\frac{1}{6} \beta_{-1}-\frac{4}{3} \beta_{2}+\frac{4}{3} \beta_{-2}-\frac{1}{6} \beta_{1}=0 \\
-\frac{1}{24} \beta_{1}-\frac{1}{24} \beta_{-1}-\frac{2}{3} \beta_{2}-\frac{2}{3} \beta_{-2}=0 \\
-2 \beta_{2}-2 \beta_{-2}-\frac{1}{2} \beta_{1}-\frac{1}{2} \beta_{-1}=0 \\
-\beta_{2}-\beta_{-2}-\beta_{1}-\beta_{-1}-\beta_{0}=0
\end{array}\right.
$$

this lead to:

$$
\begin{aligned}
\beta_{-2}=-\frac{1}{12} h^{-2}, \beta_{-1} & =\frac{4}{3} h^{-2}, \beta_{0}=-\frac{5}{2} h^{-2}, \beta_{1}=\frac{4}{3} h^{-2}, \beta_{2}=-\frac{1}{12} h^{-2} \\
\frac{\partial^{2}}{\partial x^{2}} u(t, x)= & -\frac{1}{12} \frac{u(t,-2 h+x)}{h^{2}}+\frac{4}{3} \frac{u(t,-h+x)}{h^{2}}-\frac{5}{2} \frac{u(t, x)}{h^{2}} \\
& +\frac{4}{3} \frac{u(t, h+x)}{h^{2}}-\frac{1}{12} \frac{u(t, 2 h+x)}{h^{2}}+\text { Error }
\end{aligned}
$$




$$
\begin{gathered}
\operatorname{Err}=\frac{\left(D_{2,2,2,2,2,2}\right)(u)(t, x) h^{4}}{90} \\
\frac{\partial^{2}}{\partial x^{2}} u(t, x)=-\frac{1}{12} \frac{u(t,-2 h+x)}{h^{2}}+\frac{4}{3} \frac{u(t,-h+x)}{h^{2}}-\frac{5}{2} \frac{u(t, x)}{h^{2}} \\
+\frac{4}{3} \frac{u(t, h+x)}{h^{2}}-\frac{1}{12} \frac{u(t, 2 h+x)}{h^{2}}+\frac{\left(\frac{\partial^{6}}{\partial x^{6}} u(t, x)\right) h^{4}}{90}
\end{gathered}
$$

Note that the last term on the RHS is just the leading order term in the error; terms with higher powers of have been omitted [14]. The power of $h$ in the leading error term is called the order of the stencil.

Figure 4 presents the lattice points of transonic gas equation.

Now we approximate the first derivative

$$
\frac{\partial}{\partial t} u(t, x)=\beta_{0} u(t, x)+\beta_{1} u(s+t, x)+\beta_{2} u(2 s+t, x)+\text { Error }
$$

the same method as second derivative can be done we obtain

$$
\frac{\partial}{\partial t} u(t, x)=-\frac{3}{2} \frac{u(t, x)}{s}+2 \frac{u(s+t, x)}{s}-\frac{1}{12} \frac{u(2 s+t, x)}{s}+\frac{1}{3}\left(\frac{\partial^{3}}{\partial t^{3}} u(t, x)\right) s^{2} .
$$

We will seek a solution on a rectangular region of the plane

$$
(x, y) \in[-L, L] \times[-L, L]
$$

subject to Dirichlet boundary conditions:

$$
\begin{aligned}
& u(x, y=-L)=g_{1}(x), u(x=+L, y)=g_{2}(y), \\
& u(x, y=+L)=g_{3}(x), u(x=-L, y)=g_{4}(y) \\
& \left(\frac{\partial^{2}}{\partial x^{2}} u(x, y)\right) \frac{\partial}{\partial x} u(x, y)+\frac{\partial^{2}}{\partial y^{2}} u(x, y)=0 \\
& \left(\frac{-1}{12} \frac{u(x-2 h, y)}{h^{2}}+\frac{4}{3} \frac{u(x-h, y)}{h^{2}}-\frac{5}{2} \frac{u(x, y)}{h^{2}}+\frac{4}{3} \frac{u(x+h, y)}{h^{2}} \frac{-1}{12} \frac{u(x+2 h, y)}{h^{2}}\right) \\
& \left(\frac{u(x+h, y)}{h}-\frac{u(x, y)}{h}\right)-\frac{1}{12} \frac{u(x, y-2 h)}{h^{2}}+\frac{4}{3} \frac{u(x, y-h)}{h^{2}} \\
& -\frac{5}{2} \frac{u(x, y)}{h^{2}}+\frac{4}{3} \frac{u(x, y+h)}{h^{2}} \frac{-1}{12} \frac{u(x, y+2 h)}{h^{2}}=0 \\
& {\left[\alpha_{1}\left(\frac{u_{3,2}}{h}-\frac{u_{2,2}}{h}\right)-\frac{1}{12} \frac{g_{1}\left(x_{2}\right)}{h^{2}}+\frac{4}{3} \frac{u_{2,1}}{h^{2}}-\frac{5}{2} \frac{u_{2,2}}{h^{2}}+\frac{4}{3} \frac{u_{2,3}}{h^{2}}-\frac{1}{12} \frac{u_{2,4}}{h^{2}}\right]} \\
& \alpha_{2}\left(\frac{u_{4,2}}{h}-\frac{u_{3,2}}{h}\right)-\frac{1}{12} \frac{g_{1}\left(x_{3}\right)}{h^{2}}+\frac{4}{3} \frac{u_{3,1}}{h^{2}}-\frac{5}{2} \frac{u_{3,2}}{h^{2}}+\frac{4}{3} \frac{u_{3,3}}{h^{2}}-\frac{1}{12} \frac{u_{3,4}}{h^{2}} \\
& \alpha_{3}\left(\frac{u_{3,3}}{h}-\frac{u_{2,3}}{h}\right)-\frac{1}{12} \frac{u_{2,1}}{h^{2}}+\frac{4}{3} \frac{u_{2,2}}{h^{2}}-\frac{5}{2} \frac{u_{2,3}}{h^{2}}+\frac{4}{3} \frac{u_{2,4}}{h^{2}}-\frac{1}{12} \frac{g_{3}\left(x_{2}\right)}{h^{2}} \\
& \left.\alpha_{4}\left(\frac{u_{4,3}}{h}-\frac{u_{3,3}}{h}\right)-\frac{1}{12} \frac{u_{3,1}}{h^{2}}+\frac{4}{3} \frac{u_{3,2}}{h^{2}}-\frac{5}{2} \frac{u_{3,3}}{h^{2}}+\frac{4}{3} \frac{u_{3,4}}{h^{2}}-\frac{1}{12} \frac{g_{3}\left(x_{3}\right)}{h^{2}}\right]
\end{aligned}
$$

where 


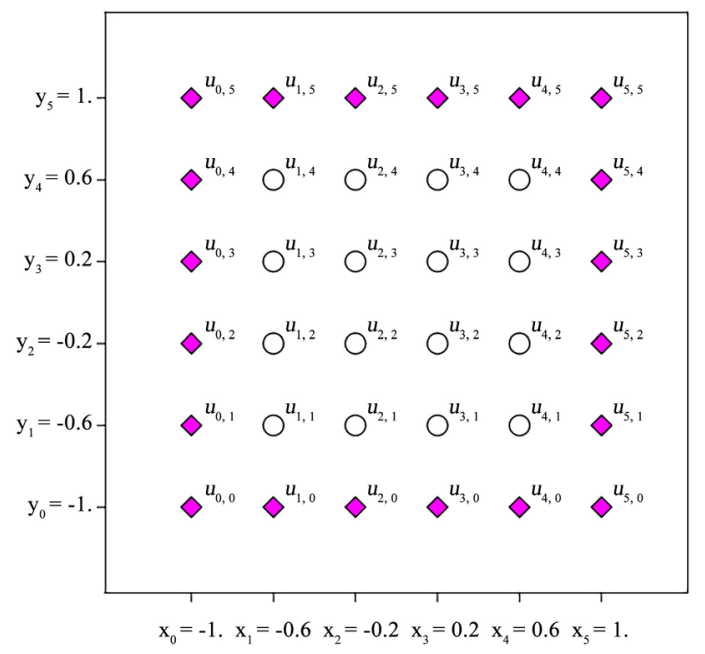

Figure 4. Lattice points solution.

$$
\begin{aligned}
& \alpha_{1}=\left(-\frac{1}{12} \frac{g_{4}\left(y_{2}\right)}{h^{2}}+\frac{4}{3} \frac{u_{1,2}}{h^{2}}-\frac{5}{2} \frac{u_{2,2}}{h^{2}}+\frac{4}{3} \frac{u_{3,2}}{h^{2}}-\frac{1}{12} \frac{u_{4,2}}{h^{2}}\right) \\
& \alpha_{2}=\left(-\frac{1}{12} \frac{u_{1,2}}{h^{2}}+\frac{4}{3} \frac{u_{2,2}}{h^{2}}-\frac{5}{2} \frac{u_{3,2}}{h^{2}}+\frac{4}{3} \frac{u_{4,2}}{h^{2}}-\frac{1}{12} \frac{g_{2}\left(y_{2}\right)}{h^{2}}\right) \\
& \alpha_{3}=\left(-\frac{1}{12} \frac{g_{4}\left(y_{3}\right)}{h^{2}}+\frac{4}{3} \frac{u_{1,3}}{h^{2}}-\frac{5}{2} \frac{u_{2,3}}{h^{2}} \frac{4}{3} \frac{u_{3,3}}{h^{2}}-\frac{1}{12} \frac{u_{4,3}}{h^{2}}\right) \\
& \alpha_{4}=\left(-\frac{1}{12} \frac{u_{1,3}}{h^{2}}+\frac{4}{3} \frac{u_{2,3}}{h^{2}}-\frac{5}{2} \frac{u_{3,3}}{h^{2}}+\frac{4}{3} \frac{u_{4,3}}{h^{2}}-\frac{1}{12} \frac{g_{2}\left(y_{3}\right)}{h^{2}}\right)
\end{aligned}
$$

Since, the number of equations is less the number of unknowns parameters, for this reason we cannot have a unique solution for our differential equation. This leads to an open question, what can we do in our case or how can we change completely the PDEs in order to get a solvable system with unique solution. Our code, will be included in the appendix, and it's available for any boundary conditions that can be introduced.

\section{Conclusion}

In this paper different methods were discussed. More precisely, we have considered a Lie group approach to get an analytical solution; this solution can be viewed as old approach that gives us a solution. To understand our problem we introduced different numerical schemes were proposed and we arrive to no unique solution that can be founded.

\section{Conflicts of Interest}

The author declares no conflicts of interest regarding the publication of this paper.

\section{References}

[1] Ames, W.F., Lohner, R.J. and Adams, E. (1981) Group Properties of $u t t=[f(u) u x] x$. 
International Journal of Non-Linear Mechanics, 16, 439-447.

https://doi.org/10.1016/0020-7462(81)90018-4

[2] Torissi, M. and Valenti, A. (1985) Group Properties and Invariant Solutions for Infinitesimal Transformations of a Non-Linear Wave Equation. International Journal of Non-Linear Mechanics, 20, 135-144. https://doi.org/10.1016/0020-7462(85)90007-1

[3] Bluman, G. and Kumei, S. (1987) Properties of the Wave Equation. Journal of Mathematical Physics, 28, 307-318. https://doi.org/10.1063/1.527659

[4] Peters, J.E. and Ames, W.F. (1990) Group Properties of the Non-Linear Dynamic Equations of Elastic String. International Journal of Non-Linear Mechanics, 25, 107-115. https://doi.org/10.1016/0020-7462(90)90042-8

[5] Fung, R.F., Wang, Y.C. and Wu, J.W. (1999) Group Properties and Group Invariant Solutions for Infinitesimal Transformations of the Non-Linearly Traveling String. International Journal of Non-Linear Mechanics, 34, 693-698. https://doi.org/10.1016/S0020-7462(98)00047-X

[6] Ozkaya, E. and Pakdemirli, M. (2000) Lie Group Theory and Analytical Solutions for the Axially Accelerating String Problem. Journal of Sound and Vibration, 230, 729-742. https://doi.org/10.1006/jsvi.1999.2651

[7] Rega, G., Srinil, N., Lacarbonara, W. and Chucheepsakul, S. (2007) Resonant Nonlinear Normal Modes of Inclined Sagged Cables. Journal of Computational and Nonlinear Dynamics, 2, 324-336.

[8] Srinil, N., Rega, G. and Chucheepsakul, S. (2004) Three-Dimensional Non-Linear Coupling and Dynamic Tension in the Large-Amplitude Free Vibrations of Arbitrarily Sagged Cables. Journal of Sound and Vibration, 269, 823-852. https://doi.org/10.1016/S0022-460X(03)00137-8

[9] Srinil, N., Chucheepsakul, S. and Rega, G. (2005) Internally Resonant Nonlinear Free Vibrations of Horizontal/Inclined Sagged Cables. ASME 2005 International Design Engineering Technical Conferences and Computers and Information in Engineering Conference, Long Beach, 24-28 September 2005, 2021-2029.

[10] Altoum, S.H. (2016) Lie Group and RK4 for Solving Nonlinear First Order ODEs. International Journal of Applied Mathematical Research, 5, 117-122. https://doi.org/10.14419/ijamr.v5i2.6033

[11] Bertschinger, E. (2002) Introduction to Tenser Calculus for General Relativity.

[12] Ibragimov, N.H. (1996) Elementary Lie Group Analysis and Ordinary Differential Equations. John Wiley Sons, New York.

[13] John, M.L. (2002) Introduction to Smooth Manifolds. Springer Verlag, Berlin.

[14] Chnitia, C., Alhazmic, S.E.A., Altouma, S.H. and Toujanid, M. (2016) DtN and NtD Surface Radiation Conditions for Two-Dimensional Acoustic Scattering: Formal Derivation and Numerical Validation. Applied Numerical Mathematics, 101, 53-70. https://doi.org/10.1016/j.apnum.2015.08.013 